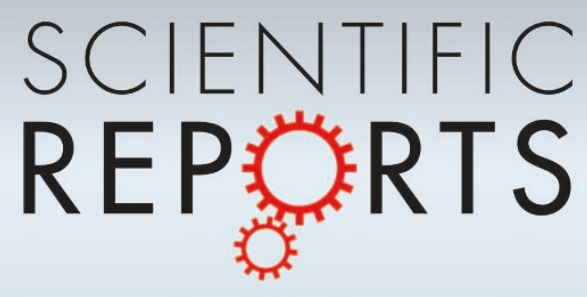

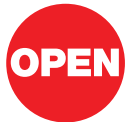

SUBJECT AREAS:

ELECTRONIC PROPERTIES AND DEVICES

SPINTRONICS

MAGNETIC DEVICES

COMPUTATIONAL SCIENCE

Received

13 August 2012

Accepted

20 February 2013

Published

5 March 2013

Correspondence and requests for materials should be addressed to

K.L.Y. (klyao@mail. hust.edu.cn)

\section{Spin Seebeck Effect and Thermal Colossal Magnetoresistance in Graphene Nanoribbon Heterojunction}

\author{
Yun $\mathrm{Ni}^{1}$, Kailun Yao',2, Huahua Fu', Guoying Gao', Sicong Zhu' \& Shuling Wang'
}

'School of Physics and Wuhan National High Magnetic Field Center, Huazhong University of Science and Technology, Wuhan
430074, China, ${ }^{2}$ International Center of Materials Physics, Chinese Academy of Science, Shenyang 110015, China.

Spin caloritronics devices are very important for future development of low-power-consumption technology. We propose a new spin caloritronics device based on zigzag graphene nanoribbon (ZGNR), which is a heterojunction consisting of single-hydrogen-terminated ZGNR (ZGNR-H) and double-hydrogen-terminated ZGNR $\left(\mathrm{ZGNR}-\mathrm{H}_{2}\right)$. We predict that spin-up and spin-down currents flowing in opposite directions can be induced by temperature difference instead of external electrical bias. The thermal spin-up current is considerably large and greatly improved compared with previous work in graphene. Moreover, the thermal colossal magnetoresistance is obtained in our research, which could be used to fabricate highly-efficient spin caloritronics MR devices.

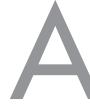

$s$ the world's increasing demand for energy is going to exhaust traditional energy, it is very important to develop new materials, which can supply sustainable and green energy for future. One kind of promising resolution lies on thermoelectric materials, which reveal the interplay of heat and charge transport ${ }^{1-3}$. As we know, spintronics, a new type of electronics, concerns the interplay of spin and charge transport $^{4-6}$. Recently, spin caloritronics, the combination of thermoelectrics and spintronics, focusing on heat and spin transport, has attracted a great attention ${ }^{7-9}$. A notable recent discovery of spin caloritronics is the observation of spin Seebeck effect by Uchida et al. ${ }^{10,11}$ They found that the spin-polarized currents of spin-up and spin-down, which flow in opposite directions, can be generated only from a temperature gradient, requiring no electrical bias. This remarkable discovery strongly promotes research on new energy of thermoelectricity ${ }^{8,9,12-16}$.

During the past a few years, Graphene, a quasi-two-dimensional crystal with exceptional electronic properties $^{17-20}$, has attracted much attention since the successful fabrication in $2004^{21}$. In particular, zigzag graphene nanoribbons (ZGNRs) are more notable for its potential applications in spintronic devices such as spin-filter ${ }^{22}$, spin-valve ${ }^{18,23}$, giant magnetoresistance devices ${ }^{24,25}$, etc. Recently, Zeng et al. found that thermally induced spinpolarized currents are generated by applying temperature difference between the source and the drain, both in a magnetized ZGNR ${ }^{9}$ and a ZGNR heterostructure ${ }^{26}$. Their results indicated the possibility of developing graphenebased spin caloritronic devices, and motivated by this, we find a new kind of graphene-based caloritronic devices. A general research of ZGNRs is passivating the edge carbon atoms with hydrogen atoms, which has different hydrogen-terminated pattern ${ }^{27-30}$. Herein we consider a heterojunction of single-hydrogen-terminated ZGNR (ZGNR-H, $\mathrm{sp}^{2}$-hybrid) and double-hydrogen-terminated ZGNR (ZGNR- $\mathrm{H}_{2}, \mathrm{sp}^{3}$-hybrid), which could be hopefully fabricated in experiment since the composition of the $\mathrm{sp}^{2}$ and $\mathrm{sp}^{3}$-like bonds at the edges might be feasible experimentally by controlling the chemical potential of hydrogen via temperature and pressure of $\mathrm{H}_{2}$ gas $^{27,31}$. Up to now, since there are only a few studies on the transport properties of graphene-based heterojunction, which are focused only on bias-induced currents ${ }^{29}$, this study becomes valuable. We found that thermally induced currents in ZGNR-H/ZGNR- $\mathrm{H}_{2}$ heterojunction are obviously enhanced compared with the former graphene-based spin caloritronic devices ${ }^{9,26}$. Furthermore, the spin Seebeck effect found in this heterojunction is caused by the structure designed and transmission spectrum. As is known, because of the Fermi-Dirac distribution, the carrier concentration gradient depends on the temperature gradient, and the asymmetric spin-polarized transmission spectrum near the Fermi energy is the essential condition for the thermally induced current. In addition, the thermal colossal magnetoresistance effect (CMR) is observed, indicating a better prospect for spin caloritronics device applications. 


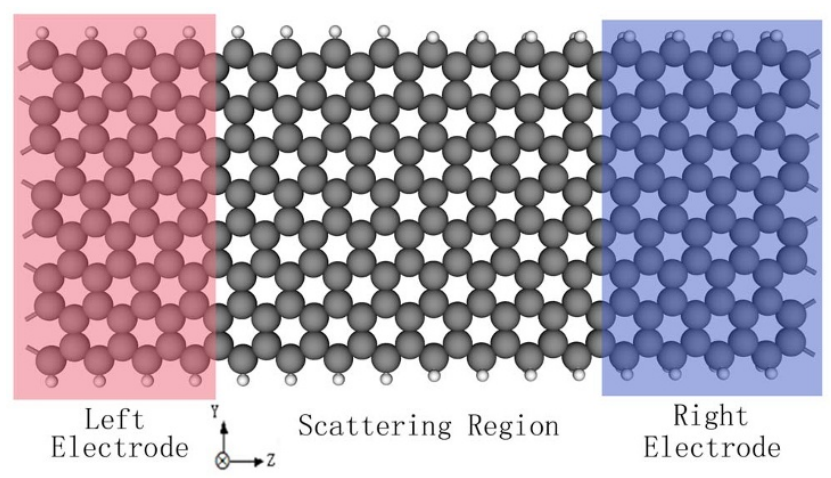

Figure 1 The schematic illustration of the thermal spin device based on ZGNR-H/ZGNR- $\mathrm{H}_{2}$ heterojunction. The spin currents are induced by $\Delta \mathrm{T}$, the temperature difference between the left electrode $\left(\mathrm{T}_{\mathrm{L}}\right)$ and the right electrode $\left(\mathrm{T}_{\mathrm{R}}\right)$, that is, $\Delta \mathrm{T}=\mathrm{T}_{\mathrm{L}}-\mathrm{T}_{\mathrm{R}}$.

\section{Results}

The heterojunction of ZGNR-H and ZGNR- $\mathrm{H}_{2}$ based on 8-ZGNR is shown in Fig. 1. As we know, the ZGNR-H has been reported having a magnetic insulating ground state with antiferromagnetic coupling of spin polarized edge states ${ }^{32}$, and can be magnetized by applying a sufficiently strong magnetic field, leading to a ferromagnetic
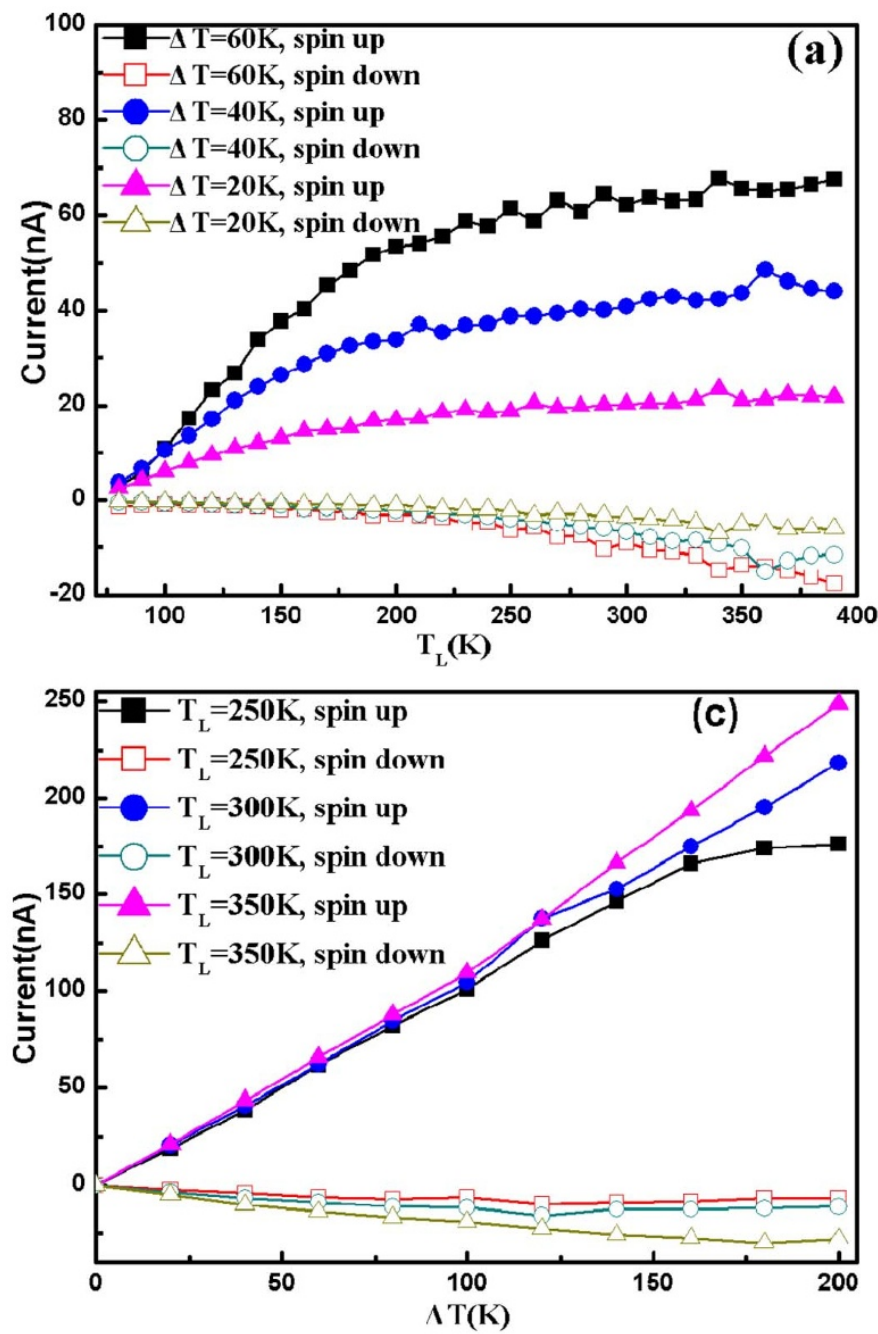

alignment of spin polarized edge states ${ }^{24,33}$. For $8 \mathrm{ZGNR}-\mathrm{H}_{2}$, we calculated the electronic band structures and the density of states (DOS) for different initial settings of spin configurations: non-magnetic, ferromagnetic, and antiferromagnetic, our numerical results show that finally the non-magnetic state is always obtained. This result is consistent with the consequence that Lee, G. et al. announced in their article ${ }^{28}$. Therefore, the magnetic state of the heterojunction can be controlled by an external magnetic field. The thermally induced currents are caused by a temperature difference $(\Delta \mathrm{T})$ between the temperature of left electrode $\left(\mathrm{T}_{\mathrm{L}}\right)$ and right electrode $\left(\mathrm{T}_{\mathrm{R}}\right)$, that is, $\Delta \mathrm{T}$ $=\mathrm{T}_{\mathrm{L}}-\mathrm{T}_{\mathrm{R}}$, without external bias voltage. Simultaneously, we choose the back gate voltage to be fixed at zero, so the carrier concentration and the spin currents are only determined by temperature. In our calculation, the transmission coefficients of the non-magnetic heterojunction are almost zero near the Fermi energy, and the thermally induced current is rather small in antiferromagnetic state. Hence, in this article we will mainly focus on ferromagnetic state of the heterojunction in upward magnetic field.

Figure 2a shows the calculated thermally induced currents versus $\mathrm{T}_{\mathrm{L}}$ of a magnetized ZGNR-H/ZGNR- $\mathrm{H}_{2}$ heterojunction at different $\Delta \mathrm{T}$. It can be clearly seen that the spin-polarized currents are generated without any transverse electric field, wherein the spin-up currents are positive while the spin-down ones are negative. This is an obvious spin Seebeck effect since the spin-up and spin-down currents flow in opposite directions, which are generated only from
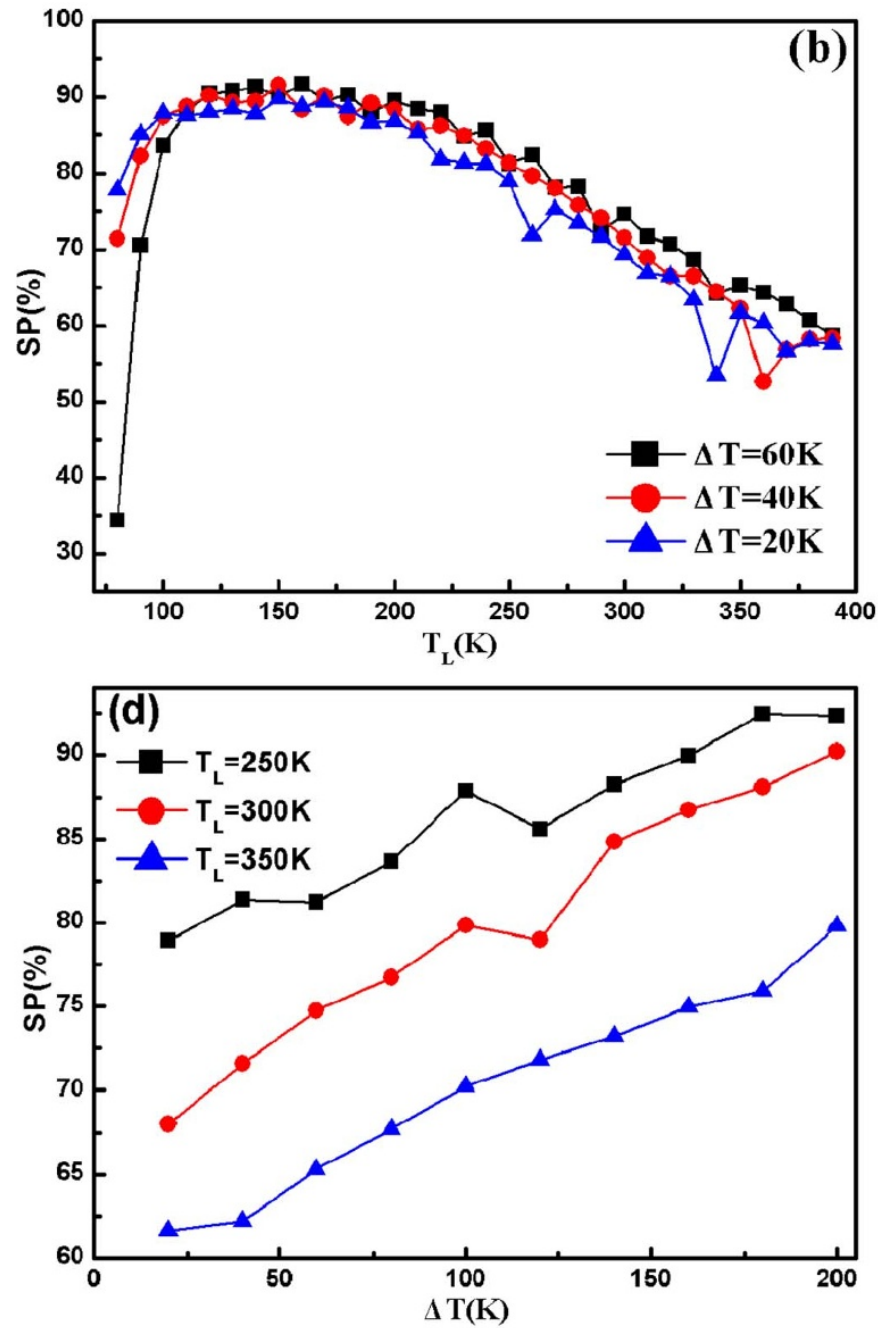

Figure $2 \mid$ The thermal spin-dependent currents versus $\mathrm{T}_{\mathrm{L}}$ (a) and versus $\Delta \mathrm{T}(\mathrm{c})$. The spin polarization as a function of $\mathrm{T}_{\mathrm{L}}(\mathrm{b})$ and as a function of $\Delta \mathrm{T}(\mathrm{d})$, calculated by $\mathrm{SP}(\%)=\left(\left|\mathrm{I}_{\text {up }}\right|-\left|\mathrm{I}_{\text {down }}\right|\right) /\left(\left|\mathrm{I}_{\text {up }}\right|+\left|\mathrm{I}_{\text {down }}\right|\right) \times 100$. 
a temperature gradient ${ }^{10,11,34}$. From the curves of spin-up currents, we find there is no threshold temperature, and the spin-up currents increase rapidly at low temperature. It can be obviously seen that the value of spin-up currents are extremely larger than the spin-down ones. The calculated spin polarizations (SPs) of the thermally induced currents reach $90 \%$ in a wide range of $\mathrm{T}_{\mathrm{L}}$, as shown in Fig. 2b. It is notable that the value of spin-up current is quite large and nearly an order of magnitude larger than previous work in graphene-based spin caloritronics without external bias voltage and gate-voltage $e^{9,26}$. In Fig. 2c, it is shown that the spin-up and spin-down currents increase as $\Delta \mathrm{T}$ increases, predictably, flowing in opposite directions. It's interesting that in most part, the currents rise almost linearly. Take spin-up one for example, when $\Delta \mathrm{T}$ is less than $160 \mathrm{~K}$ and $\mathrm{T}_{\mathrm{L}}=250 \mathrm{~K}$, the value of thermal spin-up current is proportional to $\Delta \mathrm{T}$ (as $\mathrm{I} \sim \alpha \Delta \mathrm{T}$, where $\alpha \approx 1.04 \mathrm{nA} / \mathrm{K})$. When $\mathrm{T}_{\mathrm{L}}=300 \mathrm{~K}$ and $350 \mathrm{~K}, \alpha$ is approximately $1.09 \mathrm{nA} / \mathrm{K}$ and $1.20 \mathrm{nA} / \mathrm{K}$ respectively. These results show that at room temperature, our device show obvious spin caloritronics effects, and the thermal spin-polarized current could be estimated by the temperature difference. Notice that the large temperature differences $\Delta \mathrm{T}$ displayed here is just to show the trend of the thermal current with the increase of $\Delta \mathrm{T}$, since a large $\Delta \mathrm{T}$ is not feasible experimentally in such a small device at present time. Meanwhile, as $\Delta \mathrm{T}$ increases, the spin polarization increases rapidly as well (see Figure 2d).

In addition, for the symmetry-inverted situation $\left(T_{R}>T_{L}\right)$, although our device is not a full symmetric system, the transport curves only show a slight deviation, which could be neglected in application. Moreover, we can increase spin polarization of the thermally induced currents and obtain pure spin current at room temperature by applying appropriate external transverse electrical bias of the electrodes. For example, when $\mathrm{T}_{\mathrm{L}}$ is $350 \mathrm{~K}$ and $\mathrm{T}_{\mathrm{R}}$ is $230 \mathrm{~K}$, we can obtain thermally induced spin-up current $0.24 \mu \mathrm{A}$ and nearly zero for spin-down current with $23.5 \mathrm{mV}$ transverse electrical bias and upward magnetic field.

Finally, we investigate the thermal magnetoresistance of the heterojunction changing from magnetic state (MS, external magnetic field was applied to the device) to the ground state (GS, without any external magnetic field), which can be obtained from the equation $\operatorname{MR}(\%)=\left(\mathrm{R}_{\mathrm{GS}}-\mathrm{R}_{\mathrm{MS}}\right) /\left(\mathrm{R}_{\mathrm{MS}}\right) \times 100=\left[\left(\mathrm{I}_{\mathrm{MS}}-\mathrm{I}_{\mathrm{GS}}\right) / \mathrm{I}_{\mathrm{GS}}\right] \times 100^{35}$, where $I_{M S}$ and $I_{G S}$ is the total current in the MS and GS state. Seen from Fig. 3a, as $\mathrm{T}_{\mathrm{L}}$ increases, the MS total currents increase rapidly at first, since the spin-up currents are substantially larger than the spindown ones although they flow in different directions (Figure 2a), then they remain large but slightly decline because the spin polarizations decrease at higher temperature (Figure 2b); meanwhile, the GS total currents are too small to see and just rise a little at very high temperature (Figure 3a). Therefore, the thermal magnetoresistance effect is very significant in our device. As shown in Fig. 3b, when the electronic state switches from magnetic state to ground state, the MR ratio can be tuned by $\mathrm{T}_{\mathrm{L}}$, and remains larger than $10^{6} \%$ in a wide range of temperature from $80 \mathrm{~K}$ to $360 \mathrm{~K}$, and finally drops to $10^{3} \%$ when $\mathrm{T}_{\mathrm{L}}=390 \mathrm{~K}$. We notice that the influence of $\Delta \mathrm{T}$ is not obvious at high $\mathrm{T}_{\mathrm{L}}(>250 \mathrm{~K})$ and the curve trend is almost identical. On the other hand, the MR ratio can also be tuned by $\Delta \mathrm{T}$ (Figure $3 \mathrm{c}$ ), which remains large and stable at $\mathrm{T}_{\mathrm{L}}=300 \mathrm{~K}$, the room temperature. So the thermally induced CMR of the ZGNR-H/ZGNR- $\mathrm{H}_{2}$ device can be obtained larger than $10^{6} \%$ at room-temperature in our calculations and extremely surpass the conventional metal-based MR devices, applying only a temperature gradient of the electrodes, and both transverse electric field and gate voltage are not needed. Certainly, our calculation is based on an ideal structure and limited by the validity of the Landauer framework, while a real experimental system is guaranteed to have imperfections, so the value of the thermal current and CMR would be affected by the defects. In theory, the electronic transport caused by a temperature gradient would be suppressed by the existence of defects, similar to the situation of
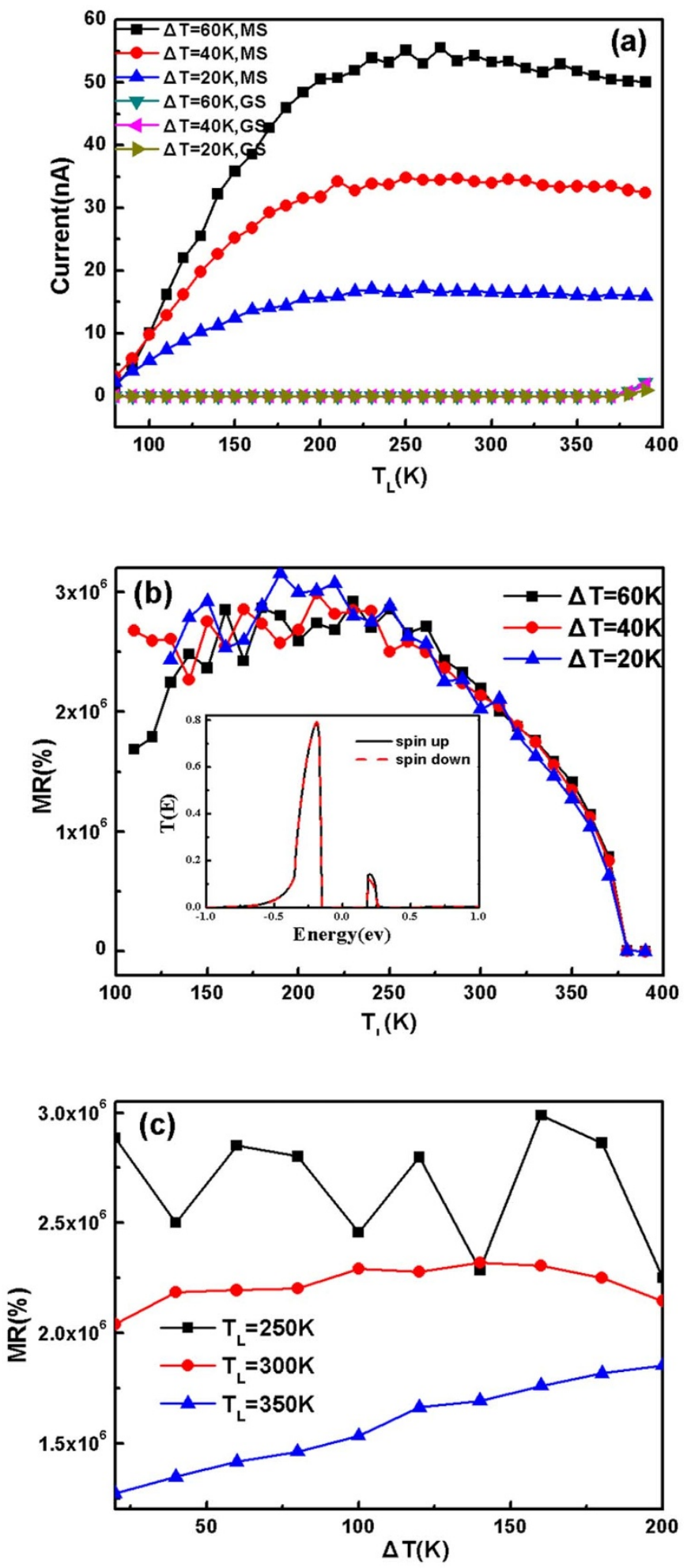

Figure 3 (a) The total spin current as a function of $T_{L}$ in magnetic state (MS) and ground state (GS) respectively. The magneto-resistance (MR) as a function of $\mathrm{T}_{\mathrm{L}}$ (b) and $\Delta \mathrm{T}$ (c). The inset of (b) shows the transmission spectrum of the heterojunction in GS state.

bias-controlled transport ${ }^{36}$. In fact, compared to most theoretical predictions, the experimental observations of MR are always much smaller $^{24,25}$. Therefore, although our results could not be taken as real values, they still have some significance for fabricating graphenebased spin caloritronics nanodevices. 


\section{Discussion}

For further insight into the interesting phenomenon described above, it is necessary to analyze the electron distribution of the left and right electrodes, which differs in the carrier concentration and is determined by the Fermi-Dirac distribution. From Eqn.(1), we know that the spin-dependent current is generated by the combined effects of transport coefficients and the difference of Fermi-Dirac distribution between left and right electrodes. First, let us take a look at the Fermi-Dirac distribution of the left and right leads with different temperatures, which is defined as $f(E, T)=\frac{1}{\exp [(E-\mu) / k T]+1}$, where $\mu$ is the chemical potential and set to zero in our discussion. From the figure of Fermi-Dirac distribution (figure 4a), we focus on two cases: the first one, carriers (electron) with energy higher than the Fermi level, flow from the left electrode to the right one, because the electron distribution of left electrode is higher than that of the right one, resulting in a negative current; the second one, carriers (hole) with energy lower than the Fermi level, flow also from the left electrode to the right one, since the electron distribution of left electrode is lower than the right one, giving rise to a positive current. If the transmission spectrum is symmetric, the current will be canceled by each other, leading to zero net current. In order to clarify the transport of the carriers, the band structures of ZGNR-H and ZGNR- $\mathrm{H}_{2}$ electrodes are calculated and presented in Fig. 4 b. One can find that only the $\pi$ and $\pi^{*}$ subbands appear near the Fermi level, either of the left and right electrodes. It has been reported that the transmission channel is open when symmetry of spin subbands of the left and right electrodes matches, and is closed when mismatches ${ }^{19,37}$. We know that under the yz midplane mirror operation, $\pi$ subband has odd parity while $\pi^{*}$ has even parity of $Z$ GNR- $\mathrm{H}^{19,24}$. However, for $\mathrm{ZGNR}-\mathrm{H}_{2}$, the parity of $\pi$ is even and $\pi^{*}$ is odd ${ }^{29}$. For spin-up transmission, the channel is open in the energy region $[-0.3$, $0 \mathrm{eV}$ ] because of symmetry match (both even parity) for the left and right electrodes, while closed in rest region because of symmetry mismatch. Simultaneously, the spin-down channel is open in the energy region $[0,0.2 \mathrm{eV}]$ and closed in the rest region, because of the symmetry too. When the Fermi-Dirac distribution is located in the asymmetric spin-polarized $\mathrm{T}(\mathrm{E})$, the spin-polarized currents are generated. It is seen from the transmission curve of middle panel in Fig. $4 \mathrm{~b}$, the transmission peak of spin-up electron appears at energies below Fermi level, and hence, carriers (hole) can transport from left electrode to the right one, leading to a positive spin-up current. Conversely, the spin-down electrons show transmission peak above Fermi level, and the transport of carriers (electron) cause a negative spin-down current from the left electrode to the right one. As a result, the ZGNR-H/ZGNR- $\mathrm{H}_{2}$ heterojunction exhibits the spin Seebeck effect. Since the peak of spin-up transport spectrum is much higher than the spin-down one and closer to Fermi level, the spin-up currents are much larger than the spin-down ones. We notice that the transmission spectra are very close to Fermi level for both spin-up and spin-down electrons, thus no temperature threshold is observed.

Now, let us turn attention to the quantitative analysis of the spin-resolved currents. The current spectra $\left(J(E)=T(E)\left(f_{L}\left(E, T_{L}\right)-\right.\right.$ $\left.\left.f_{R}\left(E, T_{R}\right)\right)\right)$ of spin-up and spin-down electrons for three different temperature configurations are shown in Fig. $4 \mathrm{c}$, where the areas covered under the curves associated with $\mathrm{X}$-axis reveal the value of current. Taking spin-up spectra for example, when $\Delta \mathrm{T}$ is fixed $(60 \mathrm{~K})$, the peak of the current spectrum at $\mathrm{T}_{\mathrm{L}}=250 \mathrm{~K}$ is higher than that at $\mathrm{T}_{\mathrm{L}}=350 \mathrm{~K}$. Nevertheless, the area covered at $\mathrm{T}_{\mathrm{L}}=250 \mathrm{~K}$ is smaller. Accordingly, the spin-up current increases as $T_{L}$ increases (Figure $2 \mathrm{a}$ ). When $\mathrm{T}_{\mathrm{L}}$ is fixed, the current spectrum and the covered area at $\Delta \mathrm{T}=100 \mathrm{~K}$ is obviously larger than $\Delta \mathrm{T}=60 \mathrm{~K}$, resulting in a substantial increasing spin-up current with the growth of $\Delta \mathrm{T}$ (Figure 2c). Likewise, the spin-down current spectra with smaller values show the same consequence with the spin-up ones.
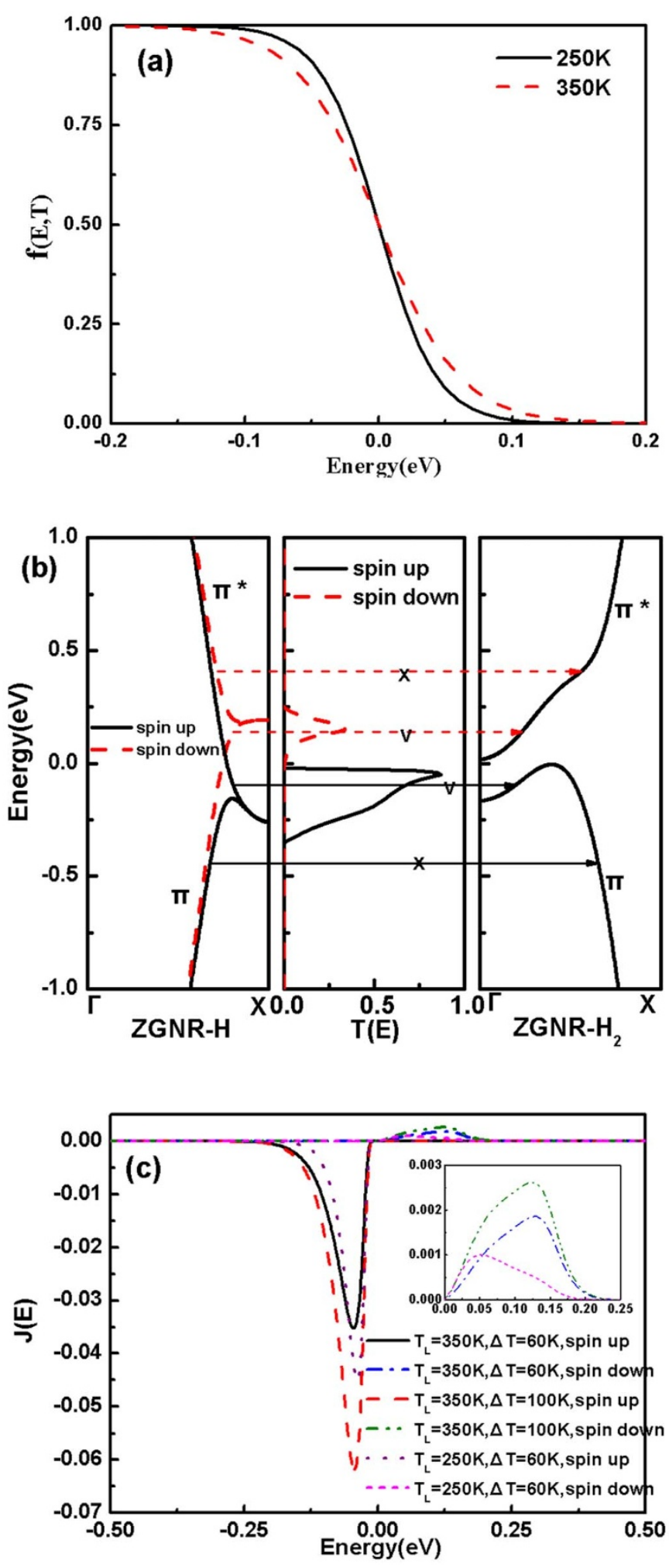

Figure $4 \mid$ (a) The Fermi distribution of electrode at different temperatures. (b) Band structure of the left electrode ZGNR-H (left panel) and the right electrode $\mathrm{ZGNR}-\mathrm{H}_{2}$ (right panel), transmission spectra (middle panel). The dashed lines marked with $\mathrm{V}(\mathrm{X})$ illustrate an allowed (forbidden) transition of electron from left electrode to the right. (c) The spin-dependent current spectra for different $T_{L}$ and $\Delta T$. The inset shows a magnified image of the spin-down current spectra. 
The origin of CMR is the huge difference between total thermal currents of $\mathrm{I}_{\mathrm{MS}}$ and $\mathrm{I}_{\mathrm{GS}}$ in the heterojunction, essentially originating from the different transmission spectra of MS and GS state. Since the temperature configuration is fixed, which means the Fermi-Dirac distributions are identical, the thermal currents of MS and GS state are just determined by transmission spectra. From transmission spectrum of GS state (inset of figure $3 \mathrm{~b}$ ), we find that the spin-up and spin-down transmission spectra nearly coincide with each other and the peaks above and below Fermi level are located at $\pm 0.2 \mathrm{ev}$. Since the transmission peak below Fermi level is higher than that above Fermi level, the positive hole current is larger than negative electron current, resulting a positive total current $\mathrm{I}_{\mathrm{GS}}$. From Fig. 4a, we know that the difference of Fermi-Dirac distribution is small when the energy is far from the Fermi level, which is extremely tiny at $\pm 0.2 \mathrm{ev}$, so $\mathrm{I}_{\mathrm{GS}}$ is considerably small (figure $3 \mathrm{a}$ ). Thus, CMR is obtained in our heterjunction, and this perfect thermal CMR effect can be employed to obtain a highly-efficient spin caloritronics device_a low-power-consumption thermal spin valve device.

\section{Methods}

Our first-principles calculations are based on the ATOMISTIX TOOLKIT (ATK) package ${ }^{38-40}$, which adopts spin density functional theory combined with nonequilibruim Green's function. The core electrons are described by norm-conserving pseudopotentials, and the local-density approximation (LDA) is used for the exchange-correlation potential. Generalized gradient approximation (GGA) exchange-correlation $(\mathrm{xc})$ functional delivered similar results, while the convergence of LDA is faster and less computation time is demanded ${ }^{41}$. A single-polarized (SZP) basis set is used and the cutoff energy is 150 Ry and a Monkhorst-Pack k-mesh of $1 \times 1 \times 100$ is chosen in our work. The spin-dependent current through the system is calculated using the Landauer formula:

$$
I^{\uparrow(\downarrow)}=\frac{e}{h} \int_{-\infty}^{\infty}\left\{T^{\uparrow(\downarrow)}(E)\left[f_{L}\left(E, T_{L}\right)-f_{R}\left(E, T_{R}\right)\right]\right\} d E,
$$

where $f_{\mathrm{L}(\mathrm{R})}(\mathrm{E}, \mathrm{T})$ is the equilibrium Fermi distribution for the left (right) electrode, and $\mathrm{T}^{\dagger(\downarrow)}(\mathrm{E})$ is the spin-resolved transmission defined as

$$
T^{\uparrow(\downarrow)}(E)=\operatorname{Tr}\left[\Gamma_{L} G^{R} \Gamma_{R} G^{A}\right]^{\uparrow(\downarrow)},
$$

where $\mathrm{G}^{\mathrm{R}(A)}$ is the retarded (advanced) Green's functions of the central region and $\Gamma_{\mathrm{L}(\mathrm{R})}$ is the coupling matrix of the left (right) electrode.

1. DiSalvo, F. J. Thermoelectric cooling and power generation. Science 285, 703-706 (1999).

2. Sales, B. C. Thermoelectric materials. Smaller is cooler. Science 295, 1248-1249 (2002).

3. Boukai, A. I. et al. Silicon nanowires as efficient thermoelectric materials. Nature 451, 168-171 (2008)

4. Wolf, S. A. et al. Spintronics: a spin-based electronics vision for the future. Science 294, 1488-1495 (2001).

5. Zhu, L., Yao, K. L. \& Liu, Z. L. Spin-polarized transport and the electronic structure of the metallic antiferromagnet Fe(thiazole)(2)Cl(2). J Chem Phys 131, 204702 (2009).

6. Cheng, H. G., Liu, Z. L. \& Yao, K. L. Rectifying behavior in La2/3Sr1/3MnO3/ $\mathrm{MgO} / \mathrm{SrRuO} 3$ magnetic tunnel junctions. Appl Phys Lett 98, 172107 (2011).

7. Bauer, G. E., Saitoh, E. \& van Wees, B. J. Spin caloritronics. Nat Mater 11, 391-399 (2012)

8. Lin, W. et al. Giant spin-dependent thermoelectric effect in magnetic tunnel junctions. Nat Commun 3, 744 (2012).

9. Zeng, M., Feng, Y. \& Liang, G. Graphene-based spin caloritronics. Nano Lett 11, 1369-1373 (2011).

10. Uchida, K. et al. Observation of the spin Seebeck effect. Nature 455, 778-781 (2008).

11. Uchida, K. et al. Spin Seebeck insulator. Nat Mater 9, 894-897 (2010).

12. Huang, S. Y., Wang, W. G., Lee, S. F., Kwo, J. \& Chien, C. L. Intrinsic spindependent thermal transport. Phys Rev Lett 107, 216604 (2011).

13. Le Breton, J. C., Sharma, S., Saito, H., Yuasa, S. \& Jansen, R. Thermal spin current from a ferromagnet to silicon by Seebeck spin tunnelling. Nature 475, 82-85 (2011).

14. Walter, M. et al. Seebeck effect in magnetic tunnel junctions. Nat Mater 10, 742-746 (2011).

15. Kirihara, A. et al. Spin-current-driven thermoelectric coating. Nat Mater. 11 686-689 (2012).

16. Weiler, M. et al. Local charge and spin currents in magnetothermal landscapes. Phys Rev Lett 108, 106602 (2012).

17. Shen, L. et al. Electron transport properties of atomic carbon nanowires between graphene electrodes. J Am Chem Soc 132, 11481-11486 (2010).
18. Zeng, M., Shen, L., Cai, Y., Sha, Z. \& Feng, Y. Perfect spin-filter and spin-valve in carbon atomic chains. Appl Phys Lett 96, 042104 (2010).

19. Zeng, M., Shen, L., Zhou, M., Zhang, C. \& Feng, Y. Graphene-based bipolar spin diode and spin transistor: Rectification and amplification of spin-polarized current. Phys Rev B 83, 115427 (2011).

20. Levy, N. et al. Strain-Induced Pseudo-Magnetic Fields Greater Than 300 Tesla in Graphene Nanobubbles. Science 329, 544-547 (2010).

21. Novoselov, K. et al. Electric field effect in atomically thin carbon films. Science 306, 666-669 (2004).

22. Nakabayashi, J., Yamamoto, D. \& Kurihara, S. Band-Selective Filter in a Zigzag Graphene Nanoribbon. Phys Rev Lett 102, 066803 (2009).

23. A Rycerz, J. T. Valley filter and valley valve in graphene. Nat Phys 3, 172-175 (2007).

24. Kim, W. Y. \& Kim, K. S. Prediction of very large values of magnetoresistance in a graphene nanoribbon device. Nat Nanotechnol 3, 408-412 (2008).

25. Bai, J. et al. Very large magnetoresistance in graphene nanoribbons. Nat Nanotechnol 5, 655-659 (2010).

26. Zeng, M., Feng, Y. \& Liang, G. Thermally induced currents in graphene-based heterostructure. Appl Phys Lett 99, 123114 (2011).

27. Wassmann, T., Seitsonen, A. P., Saitta, A. M., Lazzeri, M. \& Mauri, F. Structure, stability, edge states, and aromaticity of graphene ribbons. Phys Rev Lett 101, 96402 (2008)

28. Lee, G. \& Cho, K. Electronic structures of zigzag graphene nanoribbons with edge hydrogenation and oxidation. Phys Rev B 79, 165440 (2009).

29. Zeng, J., Chen, K. Q., He, J., Zhang, X. J. \& Sun, C. Q. Edge Hydrogenation Induced Spin-Filtering and Rectifying Behaviors in the Graphene Nanoribbon Heterojunctions. J Phys Chem C 115, 25072-25076 (2011).

30. Lu, Y. \& Feng, Y. Band-Gap Engineering with Hybrid Graphane- Graphene Nanoribbons. J Phys Chem C 113, 20841-20844 (2009).

31. Lu, Y. et al. Effects of edge passivation by hydrogen on electronic structure of armchair graphene nanoribbon and band gap engineering. Appl Phys Lett 94 , 122111 (2009).

32. Son, Y. W., Cohen, M. L. \& Louie, S. G. Energy gaps in graphene nanoribbons. Phys Rev Lett 97, 216803 (2006)

33. Son, Y. W., Cohen, M. L. \& Louie, S. G. Half-metallic graphene nanoribbons. Nature 444, 347-349 (2006).

34. Jaworski, C. et al. Observation of the spin-Seebeck effect in a ferromagnetic semiconductor. Nat Mater 9, 898-903 (2010).

35. Tsyplyatyev, O., Kashuba, O. \& Fal'ko, V. I. Thermally excited spin current and giant magnetothermopower in metals with embedded ferromagnetic nanoclusters. Phys Rev B 74, 132403 (2006).

36. Zeng, H., Leburton, J. P., Xu, Y. \& Wei, J. Defect symmetry influence on electronic transport of zigzag nanoribbons. Nanoscale Res Lett 6, 1-6 (2011).

37. Li, Z., Qian, H., Wu, J., Gu, B. L. \& Duan, W. Role of Symmetry in the Transport Properties of Graphene Nanoribbons under Bias. Phys Rev Lett 100, 206802 (2010).

38. Taylor, J., Guo, H. \& Wang, J. Ab initio modeling of quantum transport properties of molecular electronic devices. Phys Rev B 63, 245407 (2001).

39. Brandbyge, M., Mozos, J. L., Ordejon, P., Taylor, J. \& Stokbro, K. Densityfunctional method for nonequilibrium electron transport. Phys Rev B 65, 165401 (2002).

40. Soler, J. M. et al. The SIESTA method for ab initio order-N materials simulation. J Phys Condens Mat 14, 2745 (2002).

41. Yazyev, O. V. \& Helm, L. Defect-induced magnetism in graphene. Phys Rev B 75, 125408 (2007).

\section{Acknowledgments}

This work was supported by the National Natural Science Foundation of China under Grants No. 11274130, No. 11074081, No. 11004066 and No. 11274128, by the Research Foundation for the Doctoral Program of Higher Education of China under Grants No. 20090142110063 and No. 20100142120080.

\section{Author contributions}

Y.N. designed the heterojunction and wrote the manuscript. K.Y. put forward the idea and supervised the whole work. Y.N. and S.Z. carried out the numerical calculations. Y.N. and S.W. analyzed the results. H.F. and G.G. made a discussion and did calculations in the revised manuscript. All authors reviewed the manuscript.

\section{Additional information}

Competing financial interests: The authors declare no competing financial interests.

License: This work is licensed under a Creative Commons

Attribution-NonCommercial-NoDerivs 3.0 Unported License. To view a copy of this license, visit http://creativecommons.org/licenses/by-nc-nd/3.0/

How to cite this article: Ni, Y. et al. Spin Seebeck Effect and Thermal Colossal Magnetoresistance in Graphene Nanoribbon Heterojunction. Sci. Rep. 3, 1380; DOI:10.1038/srep01380 (2013). 